\title{
ACIDENTES DE TRABALHO COM MATERIAL BIOLÓGICO EM TRABALHADORES DE SERVIÇOS DE SAÚDE*
}

\author{
Manoel Carlos Arantes ${ }^{1}$, Maria do Carmo Fernandez Lourenço Haddad ${ }^{2}$, Sonia Silva Marcon ${ }^{3}$, Mariana \\ Angela Rossaneis ${ }^{4}$, Paloma de Souza Cavalcante Pissinati ${ }^{5}$, Samuel Andrade de Oliveira ${ }^{6}$
}

\begin{abstract}
RESUMO: Objetivou-se caracterizar os acidentes de trabalho com material biológico em trabalhadores de serviços de saúde. Estudo retrospectivo, quantitativo, realizado em um hospital secundário referência para o atendimento de acidentes com material biológico, localizado no norte do Paraná. Os dados foram coletados de dezembro de 2013 a junho de 2014, em 1.061 prontuários de trabalhadores da saúde e nas fichas de registro no Sistema de Informação de Agravos de Notificação, analisados por meio de estatística descritiva. Dentre os 1.061 acidentes de trabalho com material biológico, 58,1\% ocorreram com auxiliares e técnicos de enfermagem, dos quais $82,7 \%$ eram do sexo feminino. O principal material orgânico presente em $86,1 \%$ dos acidentes foi o sangue, $88,2 \%$ ocorreram por meio de exposição percutânea, $66,1 \%$ envolveram a utilização de agulhas com lúmen e $21,9 \%$ foram decorrentes do descarte inadequado de material perfurocortante. Faz-se necessário implementar medidas preventivas, direcionadas à equipe de enfermagem.

DESCRITORES: Acidentes de trabalho; Saúde do trabalhador; Exposição ocupacional; Exposição a agentes biológicos.

\section{OCCUPATIONAL ACCIDENTS WITH BIOLOGICAL MATERIAL AMONG HEALTHCARE WORKERS}

ABSTRACT: The present study aimed at characterizing occupational accidents with biological material among healthcare workers. A retrospective, quantitative study of accidents involving biological material, conducted in a secondary reference hospital located in the northeastern region of the state of Paraná. Data were collected between December 2013 and June 2014 from 1,061 medical records of health professionals and registration sheets for the Notifiable Diseases Information System, and were analyzed using descriptive statistics. Among 1,061 occupational accidents with biological material, 58.1\% occurred among nursing aides and technicians, of whom $82.7 \%$ were women. The main organic material present in $86.1 \%$ of the accidents was blood; $88.2 \%$ occurred through percutaneous exposure, $66.1 \%$ involved the use of needles with lumen, and $21.9 \%$ resulted from inappropriate disposal of sharps. It is necessary to implement preventive measures directed to the nursing team.

DESCRIPTORS: Occupational accidents; Occupational health; Occupational exposure; Exposure to biological agents.
\end{abstract}

\section{ACCIDENTES DE TRABAJO CON MATERIAL BIOLÓGICO EN TRABAJADORES DE SERVICIOS DE SALUD}

RESUMEN: Se objetivó caracterizar los accidentes laborales con material biológico en trabajadores de servicios de salud. Estudio retrospectivo, cuantitativo, realizado en hospital de referencia secundaria para atención de accidentes con material biológico, en el norte de Paraná. Datos recolectados de diciembre de 2013 a junio de 2014, de 1.061 historia clínicas de trabajadores de salud y en fichas de registro del Sistema de Información de Afecciones de Notificación, analizados por estadística descriptiva. De los 1.061 accidentes de trabajo con material biológico, 58,1\% del sucedieron a auxiliares y técnicos de enfermería, $82,7 \%$ de los cuales era de sexo femenino. El material orgánico más presente en $86,1 \%$ de los accidentes fue la sangre, $88,2 \%$ sucedieron por exposición percutánea, 66,1\% involucró utilización de agujas con lumen, y 21.9\% derivó del descarte inadecuado de material punzocortante. Resulta necesario implementar medidas de prevención, orientadas al equipo de enfermería.

DESCRIPTORES: Accidentes de Trabajo; Salud Laboral; Exposición Profesional; Exposición a Agentes Biológicos.

*Artigo extraído da dissertação intitulada: "Perfil dos acidentes com material biológico em trabalhadores de serviços de saúde". Universidade Estadual de Londrina, 2014.

${ }^{1}$ Enfermeiro. Mestre em Enfermagem. Enfermeiro na Prefeitura Municipal de Londrina. Londrina, PR, Brasil.

${ }^{2}$ Enfermeira. Doutora em Enfermagem. Docente de Enfermagem da Universidade Estadual de Londrina. Londrina, PR, Brasil. ${ }^{3}$ Enfermeira. Doutora em Enfermagem. Docente de Enfermagem da Universidade Estadual de Maringá. Maringá, PR, Brasil.

${ }^{4}$ Enfermeira. Doutora em Enfermagem. Enfermeira da Prefeitura Municipal de Rolândia. Rolândia, PR, Brasil.

${ }^{5}$ Enfermeira. Doutoranda em Enfermagem da Universidade Estadual de Maringá. Enfermeira da Prefeitura Municipal de Rolândia. Rolândia, PR, Brasil.

${ }^{6}$ Enfermeiro. Residente em Gerência de Serviços de Enfermagem na Universidade Estadual de Londrina. Londrina, PR, Brasil.

Autor Correspondente:

Paloma de Souza Cavalcante Pissinati

Universidade Estadual de Maringá

Av. Colombo, 5.790 - 87020-900 - Maringá, PR, Brasil

E-mail: cavalcanteps7@gmail.com
Recebido: 26/04/2016

Finalizado: 09/02/2017 


\section{INTRODUÇÃO}

Os acidentes de trabalho com material biológico (ATMB) constituem problema de saúde pública mundial, acarretam prejuízos econômicos e sociais, pois o trabalhador acidentado, muitas vezes, precisa se afastar das atividades produtivas. Atualmente, o Brasil ocupa a quarta posição no ranking de ocorrência de acidentes de trabalhos fatais que, em 2013, geraram custo de US\$ 7 bilhões ao país ${ }^{(1-3)}$.

Os trabalhadores da saúde são os principais envolvidos em acidentes de trabalho com material biológico. Tal realidade está relacionada, principalmente, à complexidade das atividades executadas na prestação do cuidado, das condições laborais e da baixa adesão dos indivíduos às estratégias preventivas, como o descarte adequado de materiais perfurocortantes ${ }^{(4)}$.

Ressalta-se que os acidentes de trabalho podem ocorrer por meio da exposição do indivíduo aos fluidos potencialmente contaminados, exposição percutânea por materiais perfurocortante, bem como por mucosas que apresentam rompimento da integridade. O trabalhador acidentado está exposto às diversas doenças, dentre elas a Hepatite B, Hepatite C e a Imunudeficiência Humana (HIV), além de vivenciarem alterações emocionais em virtude da preocupação com uma possível soroconversão e contaminação de seus familiares ${ }^{(2,4-5)}$.

Portanto, imediatamente após o acidente de trabalho com material biológico, o indivíduo deve receber assistência adequada ao tipo de ocorrência, sendo a instituição a responsável por encaminhá-lo ao atendimento. Faz-se necessário que o trabalhador receba as medidas profiláticas em curto período de tempo, a fim de minimizar os riscos de transmissão de agravos, como o HIV e a Hepatite $\mathrm{B}^{(5)}$.

Destaca-se que grande parte dos acidentes pode ser evitada, desde que os trabalhadores se conscientizem sobre a importância de adotar medidas de segurança, como o uso de Equipamentos de Proteção Individual (EPI) ${ }^{(6)}$. Para tanto, as instituições devem implementar medidas de educação permantente e ações que contribuam para a prestação de cuidado seguro, com a aquisição de produtos e equipamentos de qualidade ${ }^{(5)}$.

Dessa forma, conhecer as características dos acidentes de trabalho com material biológico representa importante ferramenta gerencial para o estabelecimento de medidas de melhoria das condições de trabalho e redução dos riscos ocupacionais. Além disso, fornece subsídios para que o enfermeiro, capacitado para conduzir o atendimento desses casos, identifique precocemente os profissionais com maior risco de exposição e possa planejar ações preventivas direcionadas às principais causas dessas ocorrências.

Diante disso, este estudo teve como objetivo caracterizar os acidentes de trabalho com material biológico em trabalhadores de um serviço de saúde.

\section{- MÉTODO}

Estudo retrospectivo, descritivo, de abordagem quantitativa, desenvolvido em um hospital secundário localizado no norte do Paraná, centro de referência para o atendimento de trabalhadores que sofreram acidentes de trabalho, com material biológico, nos serviços de saúde públicos ou privados dos municípios que compõem a $17^{\mathrm{a}}$ regional de saúde do estado. A área de abrangência dessa regional é constituida por 21 municípios e possui uma população estimada em 871.267 habitantes ${ }^{(7)}$.

Foram incluídos no estudo os prontuários dos trabalhadores da área da saúde que sofreram acidentes com material biológico e possuíam ficha de notificação no Sistema de Informação de Agravos e Notificação (SINAN). Excluíram-se os acidentes ocorridos em serviços de saúde com fluxos de atendimento próprios, não acompanhados no hospital de referência.

A coleta de dados foi realizada em três etapas, entre dezembro de 2013 e junho de 2014. Na primeira, as informações foram obtidas nos prontuários arquivados no hospital de referência, sendo analisados todos os casos atendidos desde o início da implantação do protocolo de atendimento aos acidentes de trabalho com material biológico, a partir de outubro de 2010 até dezembro de 2013. 
$\mathrm{Na}$ segunda etapa, coletaram-se os dados nos prontuários eletrônicos dos trabalhadores da área da saúde que sofreram acidentes de trabalho com material biológico, atendidos no Consórcio Intermunicipal de Saúde do Médio Paranapanema (CISMEPAR). As variáveis relacionavam-se aos exames médicos realizados no período de 2010 a 2013, conduta clínica e desfecho dos casos.

Na terceira etapa, os dados foram coletados por meio do Sistema de Informação de Agravos de Notificação (SINAN), obtidos no Núcleo de Atendimento ao Trabalhador da $17^{\text {a }}$ Regional de Saúde do Paraná. Identificaram-se informações complementares referentes à caracterização dos casos de acidentes de trabalho com material biológico.

Os trabalhadores da área da saúde foram categorizados de acordo com a formação em profissionais de nível superior, os quais incluíram médicos, enfermeiros e outros profissionais de saúde graduados; nível técnico, compreendendo os técnicos e auxiliares de enfermagem; e outros, que englobou nove ocupações, como auxiliares de farmácia, agentes comunitários de saúde, socorristas e outras. Os dados foram analisados no programa Statistical Package Social Science (SPSS) versão 20.0, submetidos à estatística descritiva mediante cálculo de frequências absolutas e relativas, média, mediana e desvio padrão.

O estudo teve aprovação em outubro de 2014 pelo Comitê de Ética em Pesquisa Envolvendo Seres Humanos, conforme Protocolo n ${ }^{\circ} 780.787$.

\section{RESULTADOS}

Dentre os 1.061 acidentes de trabalho com material biológico identificados no período de 2010 a 2013, 344 (32,4\%) ocorreram em 2013, seguidos por 320 (30,2\%) em 2011, 297 (28,0\%) em 2012, e 100 $(9,4 \%)$ em 2010. Dos acidentes, 877 (82,7\%), envolveram trabalhadores do sexo feminino, com média de 34 anos de idade, mediana de 32 anos e um desvio padrão de 10 anos, variando de 24 a 44 anos de idade.

Ressalta-se que $616(58,1 \%)$ trabalhadores de nível técnico foram expostos aos acidentes com material biológico, seguidos por 253 (23,8\%) profissionais de nível superior, 114 (10,7\%) auxiliares de serviços gerais e $78(7,4 \%)$ outros profissionais.

Quanto ao local de atuação dos trabalhadores acidentados, 604 (56,9\%) trabalhavam em hospitais; seguidos 138 (13,0\%) que atuavam em Unidades Básicas de Saúde (UBS); 95 (9,0\%) em clínicas odontológicas; 56 (5,3\%) em clínicas médicas especializadas e ambulatório; 27 (2,5\%) em farmácias; e 141 (13,3\%) em outros serviços de saúde.

O material orgânico predominante nos ATMB foi o sangue, presente em $914(86,1 \%)$ casos. Os trabalhadores foram expostos por meio de perfuração percutânea em 936 (88,2\%); contato com mucosas, 109 (10,3\%); e outras formas em 16 (1,5\%).

Em relação aos agentes causadores dos acidentes de trabalho com material biológico, houve predomínio de agulhas com lúmen em $701(66,1 \%)$ casos, seguidas por agulhas sem lúmen, 90 $(8,5 \%)$, lâmina/lanceta $69(6,5 \%)$, outros causadores, $172(16,2 \%)$, e em $29(2,7 \%)$ os agentes não foram identificados. Quanto às circunstâncias, o descarte de material perfurocortante teve maior frequência, $232(21,9 \%)$ casos, seguido por $220(20,7 \%)$ ocorridos durante procedimentos cirúrgicos médicos/ odontológicos, 203 (19,1\%) decorrentes da administração de medicamentos por via parenteral, 199 $(18,8 \%)$ em punções venosas e arteriais, entre outras ocorrências.

No que se refere ao uso de EPI no momento do acidente, identificou-se que apenas as luvas foram mais utilizadas pelos trabalhadores, em $662(62,4 \%)$ casos. Em contrapartida, a proteção facial e as botas não foram utilizadas em 973 (91,7\%) e 942 (88,8\%) ocorrências, como mostra a Tabela 1. 
Tabela 1 - Utilização de Equipamentos de Proteção Individual por trabalhadores de serviços de saúde momento do acidente com material biológico. Londrina, PR, Brasil, 2014

\begin{tabular}{lcccccc} 
Equipamento de Proteção Individual & \multicolumn{2}{c}{ Sim } & \multicolumn{2}{c}{$\mathbf{N a ̃ o}$} & \multicolumn{2}{c}{ Ignorado } \\
\cline { 2 - 7 } & $\mathbf{N}$ & $\mathbf{0}$ & $\mathbf{N}$ & $\mathbf{\%}$ & $\mathbf{N}$ & $\%$ \\
\hline Avental & 335 & 31,6 & 694 & 65,4 & 32 & 3 \\
\hline Botas & 83 & 7,8 & 942 & 88,8 & 36 & 3,4 \\
\hline Luvas & 662 & 62,4 & 369 & 34,8 & 30 & 2,8 \\
\hline Máscara & 184 & 17,4 & 846 & 79,7 & 31 & 2,9 \\
\hline Óculos & 154 & 14,5 & 875 & 82,5 & 32 & 3 \\
\hline Proteção Facial & 53 & 5 & 973 & 91,7 & 35 & 3,3
\end{tabular}

No momento do acidente, 956 (90,1\%) trabalhadores declararam possuir o esquema de três doses da vacina contra a hepatite B completo, 39 (3,7\%) referiram ter o esquema incompleto e em $66(6,2 \%)$ casos essa informação não constava na ficha do SINAN.

Dentre os acidentados, dois $(0,2 \%)$ apresentaram resultado sorológico reagente para o marcador anti-HIV e para o HbsAg ( antígeno de superfície do vírus da Hepatite B). Não houve sorologia reagente para o anti-HCV (Hepatite C) e somente 256 (24,1\%) possuíam imunidade contra a hepatite B, identificada por meio do anti-HBS positivo, conforme apresentado na Tabela 2.

Tabela 2 - Resultado das sorologias dos trabalhadores de serviços de saúde no momento do acidente de trabalho com material biológico. Londrina, PR, Brasil, 2014

\begin{tabular}{lcccccccc} 
Sorologia do Acidentado & \multicolumn{2}{c}{ Reagente } & \multicolumn{2}{c}{ Não Reagente } & \multicolumn{2}{c}{ Não Realizado } & \multicolumn{2}{c}{ Ignorado } \\
\cline { 2 - 10 } & $\mathbf{N}$ & $\mathbf{\%}$ & $\mathbf{N}$ & $\mathbf{\%}$ & $\mathbf{N}$ & $\mathbf{\%}$ & $\mathbf{N}$ & $\%$ \\
\hline Anti-HIV & 2 & 0,2 & 576 & 54,3 & 30 & 2,8 & 453 & 42,7 \\
\hline HbsAg & 2 & 0,2 & 546 & 51,5 & 31 & 2,9 & 482 & 45,4 \\
\hline Anti-HBS & 256 & 24,1 & 305 & 28,7 & 35 & 3,3 & 465 & 43,9 \\
\hline Anti-HCV & - & - & 544 & 51,3 & 36 & 3,4 & 481 & 45,3
\end{tabular}

O paciente fonte era conhecido em 825 (77,8\%) acidentes registrados. Dentre esses casos, 21 (2\%) possuíam sorologia reagente para o marcador anti-HIV, $15(1,4 \%)$ para o HbsAg e $07(0,7 \%)$ para o antiHCV, de acordo com os dados apresentados na Tabela 3.

Tabela 3 - Resultados das sorologias do paciente fonte no momento do acidente de trabalho com material biológico. Londrina, PR, Brasil, 2014

\begin{tabular}{lcccccccc} 
Sorologia do & Paciente & \multicolumn{2}{c}{ Reagente } & \multicolumn{2}{c}{ Não Reagente } & \multicolumn{2}{c}{ Não Realizado } & \multicolumn{2}{c}{ Ignorado } \\
\cline { 2 - 10 } & $\mathbf{N}$ & $\mathbf{\%}$ & $\mathbf{N}$ & $\mathbf{\%}$ & $\mathbf{N}$ & $\mathbf{\%}$ & $\mathbf{N}$ & $\%$ \\
\hline Anti-HIV & 21 & 2 & 765 & 72,1 & 258 & 24,3 & 17 & 1,6 \\
\hline HbsAg & 15 & 1,4 & 769 & 72,5 & 258 & 24,3 & 19 & 1,8 \\
\hline Anti-HBS & - & - & 36 & 3,4 & 1008 & 95 & 17 & 1,6 \\
\hline Anti-HCV & 7 & 0,7 & 773 & 72,9 & 264 & 24,8 & 17 & 1,6
\end{tabular}

As sorologias das fontes para $\mathrm{HbsAg}$, anti-HIV e anti-HCV foram desconhecidas (inconclusivas, não realizadas ou ignoradas) em 278 (26,2\%) casos, e em 1025 (96,6\%) para anti-HBS.

No que se refere à conduta clínica após o acidente, a vacina contra hepatite B foi indicada em 16 $(1,5 \%)$ casos. Foi administrado imunoglobulina humana anti-hepatite B em três $(0,3 \%)$ dos acidentados. 
Além disso, houve prescrição de profilaxia com medicação antirretroviral em 268 (25,3\%) ATMB, sendo que em dois $(0,2 \%)$ casos o acidentado recusou a medicação. A combinação de escolha em 267 $(99,6 \%)$ dos casos foi por AZT+3TC+Kaletra.

Em relação ao desfecho dos casos, houve abandono do tratamento após consulta ambulatorial com especialista em 306 (28,8\%) casos. Ainda, 353 (33,3\%) tiveram alta do tratamento após confirmar que o paciente-fonte não possuía doenças transmissíveis, conforme tabela 4.

Tabela 4 - Desfechos dos casos de acidentes de trabalho com material biológico em trabalhadores de serviços de saúde. Londrina, PR, Brasil, 2014

\begin{tabular}{lcc} 
Desfecho & $\mathbf{N}$ & $\mathbf{\%}$ \\
\hline Abandono pós consulta ambulatorial & 306 & 28,8 \\
\hline Abandono sem consulta ambulatorial & 211 & 19,9 \\
\hline Alta paciente-fonte negativo & 353 & 33,3 \\
\hline Alta sem soroconversão sorológica & 103 & 9,7 \\
\hline Ignorado & 88 & 8,3
\end{tabular}

\section{- DISCUSSÃO}

A maioria dos trabalhadores era jovem e do sexo feminino. Resultado que se assemelha a outros estudos sobre $\mathrm{ATMB}^{(8-9)}$, bem como aos dados expostos em pesquisa realizada pelo Conselho Federal de Enfermagem, em parceria com a Fundação Oswaldo Cruz, que apresentou o perfil da enfermagem no Brasil, sendo identificado que, apesar da tendência a masculinização, as mulheres ainda representam $84,6 \%$ dos profissionais da área ${ }^{(10)}$.

Os profissionais de nível médio, os quais incluíam os técnicos e auxiliares de enfermagem, apresentaram maior envolvimento em acidentes de trabalho com material biológico. A equipe de enfermagem constitui o grupo de profissionais com maior tempo de permanência nos serviços de saúde e devido ao grande número de procedimentos executados, encontra-se mais vulnerável à exposição ao material biológico, com maiores riscos de se envolverem em acidentes e adquirirem doenças infecciosas ${ }^{(11)}$.

Estudo realizado no município de Teresina, Piauí, envolvendo 30 estabelecimentos de saúde, demonstrou que os profissionais da equipe de enfermagem foram os que mais sofreram acidentes de trabalho(4). Outra pesquisa realizada em serviços de referência de um município paulista, também identificou essa categoria profissional como a principal envolvida em casos de acidentes com material biológico ${ }^{(12)}$.

No presente estudo, o ambiente hospitalar foi o principal local de ocorrência dos acidentes de trabalho com material biológico, resultado que pode estar associado ao fato de se tratar de um ambiente onde se desenvolvem atividades complexas e no qual há maior contato com procedimentos invasivos. Portanto, se faz necessária a adoção de medidas especiais para a proteção dos trabalhadores, sendo a biossegurança uma forma de intervir na redução dos riscos existentes, aplicada por meio de ações educativas e preventivas, com o objetivo de minimizar a ocorrência de acidentes entre os profissionais $^{(13)}$.

O sangue foi o material orgânico de maior prevalência nos acidentes em estudo, os quais foram causados, principalmente, pelo uso de agulhas com lúmen, por meio da exposição percutânea. Esses resultados assemelham-se à literatura e podem estar relacionados à complexidade e ao elevado número de procedimentos invasivos realizados nos serviços de saúde ${ }^{(14-15)}$.

Ressalta-se que as agulhas com lúmen são grandes causadoras dos ATMB, portanto, as instituições de saúde devem disponibilizá-las com dispositivos de segurança e capacitar os trabalhadores quanto a sua utilização e descarte corretos, de acordo com a NR $32^{(16)}$. Além disso, há a necessidade de realizar 
ações de educação permanente, adequar e organizar o processo de trabalho, e, sobretudo, sensibilizar os trabalhadores quanto à importância da adoção de práticas seguras ${ }^{(17)}$.

Apesar do descarte de material perfurocortante ter constituído a principal circunstância dos ATMB no presente estudo, esta pode diferir de acordo com as características da instituição e do processo de trabalho. Alguns estudos apresentaram resultados semelhantes ${ }^{(3,18)}$, enquanto outros apontaram que os incidentes ocorreram durante a realização de procedimentos cirúrgicos, odontológicos e laboratoriais, seguidos por administração de medicação por via parenteral, recapagem de agulha, punção venosa e administração de medicamentos ${ }^{(10,12,19-20)}$.

Diante disso, reforça-se a importância dos Equipamentos de Proteção Individual na prevenção dos ATMB, os quais devem ser utilizados de acordo com os tipos de atividade desenvolvida. As luvas protegem a pele íntegra ou não íntegra do trabalhador da exposição a material biológico; os óculos e a máscara impedem o contato das mucosas ocular e bucal com os fluídos orgânicos; já, o protetor facial é indicado durante a limpeza mecânica de instrumentais; o avental confere proteção à roupa e à pele do trabalhador, enquanto as botas cobrem a pele do profissional em locais úmidos e com materiais infectantes ${ }^{(21)}$.

Apesar de sua relevância, identificou-se baixa utilização dos EPI pelos trabalhadores acidentados. Pesquisas com profissionais da saúde indicam que as maiores dificuldades quanto ao uso de EPI estão relacionadas a dificuldades que os trabalhadores enfrentam para manusear materiais e realizar procedimentos usando alguns tipos de EPI, como a luva, e, ainda, os trabalhadores referem que em situações de emergência não tem tempo de fazer o uso adequado de $\mathrm{EPI}^{(22)}$.

Outras medidas devem ser adotadas pelos trabalhadores para evitar infecções por patógenos após os ATMB, como manter o esquema vacinal contra hepatite B atualizado. Nesta pesquisa se evidenciou grande número de trabalhadores $(90,1 \%)$ que declaram estar com esquema vacinal completo, o resultado ainda está abaixo do percentual de $95 \%$ de vacinação preconizado pelo Ministério da Saúde ${ }^{(23)}$.

Ainda não existe vacina contra a hepatite $C$, mas essa doença não deve ser ignorada. As precauçõespadrão são importantes na prevenção das hepatites virais, contudo faz-se necessário conhecer o resultado da sorologia anti-HCV, para se iniciar o tratamento, quando necessário, o mais precoce o possível $^{(23)}$.

Os ATMB trazem consequências não somente à saúde do trabalhador, mas para as instituições de trabalho, já que, em alguns casos, há a necessidade de afastamento, devido ao uso de medicamentos quimioprofiláticos e as repercussões psíquicas e emocionais ${ }^{(17)}$. Portanto, o acompanhamento desse trabalhador torna-se fundamental, visto que muitos abandonam o tratamento após início da profilaxia com antirretroviral, em decorrência dos efeitos adversos ${ }^{(24)}$.

De acordo com estudo, de abordagem qualitativa, realizado em hospital de alta complexidade do estado de São Paulo, os profissionais de enfermagem apresentavam sentimentos de medo, culpa e desespero após o acidente, principalmente, pela possibilidade de infecção pelos vírus da hepatite B (HBV), hepatite C (HCV) e pelo vírus da imunodeficiência humana (HIV) ${ }^{(5)}$. Assim, para evitar o abandono do acompanhamento, é necessário implementar estratégias para aumentar a adesão dos trabalhadores, dentre as quais se destacam a conscientização e a sensibilização desde o momento do acidente, até a alta médica, independentemente de a sorologia do paciente-fonte ser não reagente.

Destaca-se como limitação deste estudo o preenchimento incompleto da ficha do SINAN, bem como o elevado índice de abandono do tratamento, o que impossibilita analisar os desfechos dos ATBM.

\section{- CONCLUSÃO}

Os acidentes de trabalho com material biológico foram frequentes entre trabalhadores em idade produtiva e em indivíduos do sexo feminino. Os profissionais acometidos pelos ATMB foram os auxiliares e técnicos de enfermagem, resultado que reforça a importância de se investir em medidas preventivas, por se tratar de indivíduos diretamente envolvidos com a assistência ao paciente. 
O sangue foi o principal material orgânico encontrado nos casos e a principal causa de acidente foi o descarte de material perfurocortante em locais inadequados, provocado, sobretudo, por agulhas com lúmen. Situação que pode estar relacionada a pouca utilização dos Equipamentos de Proteção Individual pelos trabalhadores, sendo necessária maior vigilância das instituições de saúde, por meio da implementação de ações educativas para orientá-los quanto à sua importância.

Observou-se significativo abandono do tratamento pelos trabalhadores que tiveram ATMB. Portanto, faz-se necessário estudos com vistas a compreender os motivos que levam os indivíduos a abandonarem o tratamento após o acidente, de forma a contribuir para a readequação e desenvolvimento de estratégias efetivas para a melhoria da adesão terapêutica.

\section{REFERÊNCIAS}

1. Brasil. Portal Brasil. Brasil e Alemanha discutem impacto dos acidentes de trabalho. [Internet] Brasília: MTE; 2014 [acesso em 21 abr 2016]. Disponível: http://www.brasil.gov.br/economia-e-emprego/2014/03/brasil-e-alemanhadiscutem-impacto-dos-acidentes-de-trabalho

2. Giancotti GM, Haeffner R, Solheid NLS, Miranda FMD, Sarquis LMM. Caracterização das vítimas e dos acidentes de trabalho com material biológico atendidas em um hospital público do Paraná, 2012. Epidemiol. Serv. Saúde. [Internet] 2014;23(2) [acesso em 16 jun 2016]. Disponível: http:/dx.doi.org/10.5123/S1679-49742014000200015

3. Julio RS, Filardi MBS, Marziale MHP. Work accidents with biological material occurred in municipalities of Minas Gerais. Rev. bras. enferm. [Internet] 2014;67(1) [acesso em 16 jun 2016]. Disponível: http://dx.doi.org/10.5935/00347167.20140016

4. Santos SS, da Costa NA, Mascarenhas MDM. Caracterização das exposições ocupacionais a material biológico entre trabalhadores de hospitais no Município de Teresina, Estado do Piauí, Brasil, 2007 a 2011. Epidemiol. Serv. Saúde. [Internet] 2013;22(1) [acesso 16 jun 2016]. Disponível: http://dx.doi.org/10.5123/S1679-49742013000100017

5. Magagnini MAM, Rocha SA, Ayres JA. O significado do acidente de trabalho com material biológico para os profissionais de enfermagem. Rev. gauch. enferm. [Internet] 2011;32(2) [acesso em 16 jun 2016]. Disponível: http:// dx.doi.org/10.1590/S1983-14472011000200013

6. de Lima BFR, Waffae MC, de Figueiredo EN, Filipinni R, Luz MCB, Azzalis LA, et al. Infecção ocupacional pelo vírus da hepatite B: riscos e medidas de prevenção. Rev. bras. crescimento desenvolv. hum. [Internet] 2013;23(2) [acesso 16 jun 2016]. Disponível: http://www.revistas.usp.br/jhgd/article/view/61294/64233

7. Instituto Brasileiro de Geografia e Estatística (IBGE). Cidades@. [Internet] 2010 [acesso em 16 jun 2016]. Disponível: http://www.cidades.ibge.gov.br/xtras/home.php

8. Valim MD, Marziale MHP. Avaliação da exposição ocupacional a material biológico em serviços de saúde. Texto Contexto Enferm. [Internet] 2011;20(n. esp) [acesso em 16 jun 2016]. Disponível: http://dx.doi.org/10.1590/ S0104-07072011000500018

9. Vieira M, Padilha MI, Pinheiro RDC. Analysis of accidents with organic material in health workers. Rev. LatinoAm. Enfermagem. [Internet] 2011;15(2) [acesso em 16 jun 2016]. Disponível: http://dx.doi.org/10.1590/S010411692011000200015

10. Conselho Federal de Enfermagem (COFEN). Pesquisa inédita traça perfil da enfermagem no Brasil. [Internet] 2015 [acesso em 16 jun 2016]. Disponível: http://www.cofen.gov.br/pesquisa-inedita-traca-perfil-daenfermagem_31258.html

11. Cavalcante CAA, Cavalcante EFO, Macêdo MLAF, Cavalcante ES, Medeiros SM. Acidentes com material biológico em trabalhadores. Rev. Rene [Internet] 2013;14(5) [acesso em 16 jun 2016]. Disponível: http://www. revistarene.ufc.br/revista/index.php/revista/article/viewFile/1267/pdf

12. Dias MAC, Machado AA, Santos BMO. Acidentes ocupacionais com exposição a material biológico: retrato de uma realidade. Medicina (Ribeirão Preto). [Internet] 2012;45(1) [acesso em 16 jun 2016]. Disponível: http://dx.doi. org/10.11606/issn.2176-7262.v45i1p12-22 
13. Sousa LPT, da Silva MA. Produção científica da enfermagem sobre acidentes com material biológico. Estudos, Goiânia [Internet] 2014;41(n. esp) [acesso em 16 jun 2016]. Disponível: http://dx.doi.org/10.18224/est.v41i0.3809

14. de Araújo TM, Caetano JA, Barros LV, Lima ACF, da Costa RM, Monteiro VA. Acidentes de trabalho com exposição a material biológico entre os profissionais de Enfermagem. Rev. Enf. Ref. [Internet] 2012;serlII(7): [acesso em 16 jun 2016]. Disponível: http://dx.doi.org/10.12707/RIII1182

15. Marziale MHP, Rocha FLR, Robazzi MLCC, Cenzi CM, dos Santos HEC, Trovó MEM. Organizational influence on the occurrence of work accidents involving exposure to biological material. Rev. Latino-Am. Enfermagem [Internet] 2013;21(n esp) [acesso em 16 jun 2016]. Disponível: http://dx.doi.org/10.1590/S0104-11692013000700025

16. Ministério do Trabalho e Emprego (BR). Portaria n. 1.748, de 30 de agosto de 2011. Norma Regulamentadora 32. Dispõe sobre a Segurança e Saúde no Trabalho em Serviços de Saúde. Diário Oficial da União, [Internet] 31 ago 2011 [acesso em 16 jun 2016]. Disponível: http://www.trtsp.jus.br/geral/tribunal2/ORGAOS/MTE/Portaria/ P1748_11.html

17. Oliveira JS, Nery AA, Morais RLGL, Robazzi MLCC. Acidentes com perfurocortante entre trabalhadores de saúde. Rev. APS. [Internet] 2015;18(1) [acesso em 16 jun 2016]. Disponível: http://aps.ufjf.emnuvens.com.br/aps/ article/view/2392

18. Soares LG, Sarquis LMM, Kirchhof ALC, Felli VLA. Multi-causality in nursing work accidents with biological material. Rev. bras. enferm. [Internet] 2013;66(6) [acesso em 16 jun 2016]. Disponível: http://dx.doi.org/10.1590/ S0034-71672013000600007

19. de Lima LM, de Oliveira CC, de Rodrigues, KMR. Exposição ocupacional por material biológico no hospital santa casa de pelotas - 2004 a 2008. Esc. Anna Nery. [Internet] 2011;15(1) [acesso em 16 jun 2016]. Disponível: http://dx.doi.org/10.1590/S1414-81452011000100014

20. Marziale MHP, dos Santos HEC, Cenzi CM, Rocha FLR, Trovó MEM. Consequências da exposição ocupacional a material biológico entre trabalhadores de um hospital universitário. Esc. Anna Nery [Internet] 2014;18(1) [acesso em 16 jun 2016]. Disponível: http://dx.doi.org/10.5935/1414-8145.20140002

21. Ministério da Saúde (BR). Hospital Federal de Bonsucesso. Comissão de Controle de Infecção Hospitalar. Rotina A2. Equipamento de proteção Individual (EPI) na prevenção do risco biológico e químico na área da saúde. Rio de Janeiro; 2010 [acesso em 16 jun 2016]. Disponível: http://www.hgb.rj.saude.gov.br/ccih/pag1.asp

22. Rondon EC, Tavares MS, dos Santos WL. Fatores dificultadores e facilitadores que os profissionais de enfermagem enfrentam relacionados ao uso de EPI's. Revista Gestão \& Saúde. [Internet] 2012;3(3) [acesso em 16 jun 2016]. Disponível: http://gestaoesaude.unb.br/index.php/gestaoesaude/article/view/213

23. Jardim EMA, Carvalho PAM, da Silva RP, de Souza, AC. Vacinação contra hepatite e resposta vacinal em trabalhadores da área da saúde envolvidos em acidentes com material biológico. Acta de Ciências e Saúde [Internet] 2013;2(2) [acesso em 16 jun 2016]. Disponível: http://www.Is.edu.br/actacs/index.php/ACTA/article/ view/58/64

24. Pimenta FR, Pimenta FR, Ferreira MD, Gir E, Hayashida M, Canini SRMS. Care and specialized clinical followup of nursing professionals who have been victims of accidents with biological material. Rev. Esc. Enferm. USP [Internet] 2013;47(1) [acesso em 16 jun 2016]. Disponível: http://dx.doi.org/10.1590/S0080-62342013000100025 